\title{
Concept-based and Fuzzy Adaptive E-learning
}

\author{
Mesfer Al Duhayyim \\ University of Sussex \\ Sussex House, Falmer \\ Brighton, UK, BN1 9RH \\ m.al-duhayyim@sussex.ac.uk
}

\author{
Paul Newbury \\ University of Sussex \\ Sussex House, Falmer \\ Brighton, UK, BN1 9RH \\ p.newbury@sussex.ac.uk
}

\begin{abstract}
This study aims to test an effective adaptive e-learning system that uses a coloured concept map to show the learner's knowledge level for each concept in the topic. A fuzzy logic system is used to evaluate the learner's knowledge level for each concept in the domain and produce a ranked concept list of learning materials to address weaknesses in the learner's understanding. This system will obtain information on a learner's understanding of concepts by an initial pre-test before the system is used for learning and a posttest after using the learning system. A fuzzy logic system is used to produce a weighted concept map during the learning process. The aim of this research is to prove that such a proposed novel adapted e-learning system will enhance a learner's performance and understanding. In addition, this research aims to increase participants' overall learning level and effectiveness by providing a coloured concept map of understanding followed by a ranked concepts list of learning materials.
\end{abstract}

\section{Keywords}

Adaptive E-learning System, coloured concept map, fuzzy logic, ranked concept list.

\section{INTRODUCTION}

E-learning is commonly used for web-based instruction to allow learners to access online courses through the internet [1], [2]. The benefits of e-learning systems have been addressed in many previous investigations [3]; however, there are several limitations of existing e-learning systems. Key issues with traditional e-learning are that each learner views the same learning content and is provided with the same learning style; there can be a lack of feedback and tutor interaction in the learning process [4]. The lack of feedback is one of the critical e-learning issues that could limit the user's learning. In the worst cases, this limitation can make the learners stop using the e-learning system or even drop the course [5]. The lack of tutor interaction is another critical issue in e-learning systems, where the students may have different knowledge levels or preferred learning styles [3]. Researchers in computer-based education have become increasingly interested in the subject of adaptation in e-learning and two important terms have emerged: adaptivity; and adaptability. Adaptivity describes the modification of e-learning lessons in response to user behaviour, such modification being based on what amounts to an AI application using rules that are predefined and connect with a series of different parameters. Adaptability, on the other hand, is what happens when it is the user who modifies and changes the learning process by personalising e-learning lessons to suit themselves [6], [7]. The current stage of development is that adaptation in e-learning makes use of new technologies and modes of expression developed originally in Computer Based Training and Adaptive Hypermedia Systems [7].

Various adaptive techniques have been used in adaptive e-learning systems in different forms to provide adaptation services to the e- learning environment. Adaptation e-learning techniques answer three fundamental questions in adaptive e-learning systems: What can they adapt? To what can they adapt? How can they adapt? They have been used in different aspects of e-learning environment to provide adaptivity and personalization. For example, some researchers have used adaptive techniques to determine the knowledge level of an individual student. Others have used an adaptive mechanism to identify the learning styles and preferences of each learner. Adaptive algorithms have been used to evaluate and adapt the learning content for each student based on his/her performance. Therefore, many adaptive techniques have been employed in the last decade, such as concept maps, fuzzy logic, expert system, Bayesian network, overlay model, stereotypes, big data techniques etc. to provide an effective adaptation system in the learning process [8], [9], [10], [11], [12], [13], [14].

In this paper, concept maps and fuzzy logic are used as adaptive techniques which are applied to an e-learning system to achieve this adaptivity. A concept map is a method of denoting relationships between ideas, images, or words. There are many uses of concept maps, such as stimulating the generation of ideas, brainstorming, communicating complex ideas [8]. Concept maps are used in the field of education, for instance, in assessing learner knowledge of learning goals, concepts, and the relationship between those concepts. There are two key uses of concept maps. For instance, they are used to show the structure of learning materials in a hierarchal style [15], and they are also used to capture the understanding of the student of those materials.

The concept map has been used in many research projects to help the students learn and motivate them when tackling challenging topics, such as engineering drawing [16]. The findings of this piece of research showed that the concept maps helped the students to understand how to correspond the shapes to the appropriate sides of a Socket Head Screw, they could improve their work by using 3-D animations with immediate step-by-step instructions and apply it to students with both low spatial ability and high spatial-ability. A concept map (CM) has been used to assist learners' understanding and achievement by constructing an automatically personalised concept map [17]. They have developed a concept map-smart extraction and explicit diagnosis (CM-SEED) to find learning difficulties and misunderstandings and to provide appropriate recommendations and guidance for remedial learning. Although they have positive results in their experiment, they need to apply the CMSEED learning system to more platforms and different courses to test the effectiveness of this system. Others have used the concept map to calculate the student's knowledge about a specific topic automatically [18]. However, they have mentioned that due to the large number of students who have used their system, the system needs improvement, and needs a powerful tool to handle any number of data usage. An adaptive intelligent system called OPCOMITS has been developed based on a concept map model to organise the topic in the hierarchal approach to measure the student's knowledge about a topic and to animate learning [15]. The experiment gained positive 
results for a computer programming course, but it is not clear if it would work for various other kinds of subject matter, such as sciences, humanities, and foreign languages courses. Concept maps work well with programming courses because they present any hierarchical learning material with prerequisite relationships between the concepts e.g. classes, methods, inheritance, etc. in a visual style that can simply be examined and shared. The research mentions improvements to this investigation are needed by using data mining techniques such as Bayesian Networks, fuzzy logic theory, decision tree, etc. to perform enhancements by identifying the prerequisite relationship between the concepts. By using these adaptive techniques, the experts of the course can add the suitable content based on the student's performance and arrange the concepts and the relationships between them.

In this paper, instead of using the weight of relationships between the concepts and question [15], the concept weights itself is used, and the prerequisite concepts are based on the concept impact value after the students take the pre or post-tests during the learning process.

A fuzzy logic system is used in combination with a pre-test to evaluate the student's knowledge level for each concept in the domain, and produce a ranked concept list of learning materials to address weaknesses in the learners understanding. Fuzzy logic was introduced by [19] as a method for computing with words [20]. Fuzzy logic is a design of multi-valued logic that enables intermediate values between regular evaluations such as true/false, yes/no, high/low, and big/small. Ideas such as rather tall or very fast can be declared mathematically and treated by computers to apply a more human-like approach of thinking in computer programming $[21,22]$. Fuzzy logic theory labels reasoning in the circumstances of real-life uncertainty. For example, the phrase "only slightly understood" may be represented as a score, e.g., between 65 and 75 as well as the phrase "understood fairly well" could represent the score between 80 and 90 in the field of learning environment [23, 24].

Many researchers have used fuzzy logic to determine and update the student's knowledge level model for each domain concept [25], [26], [27], [28], and to personalise and recommend a learning path to be followed by the students based on their learning evaluation [9], [29]. In particular, [25] have used fuzzy logic integrated into the student model, and four fuzzy sets for defining student knowledge of a domain concept have been identified as: Unknown (Un): the degree of progress in the domain concept is from $0 \%$ to $60 \%$. Unsatisfactory Known (UK): the degree of achievement in the domain concept is from $55 \%$ to $75 \%$. Known (K): the degree of success in the domain concept is from $70 \%$ to $90 \%$. Learned (L): the degree of success in the domain concept is from $85 \%$ to $100 \%$. They didn't justify the percentages of the degree of the achievement in the domain concept especially with "Unknown" notation and this seems higher than would normally be expected. [26] have developed the PeRSIVA system to attain validity, accuracy, and effectiveness of the intelligent e-learning system. This evaluation method is a combination of some algorithms such as the well-known Kirkpatrick assessment methodology [30] and a layered evaluation framework to design and correct the assessment process of the student model in an adaptive e-learning system. The PeRSIVA method evaluates the student model by combining fuzzy logic and stereotypes techniques of an e-learning system. They have piloted an experiment on a group of students who used the system to learn the programming language C. The results of the experiment were considered satisfactory and it helped them with adaptivity to make valid decisions. However, they have suggested making the PeRSIVA framework more valid; it has to be examined in various learning circumstances, such as other courses types; data structures, database systems.

[29] have developed an educational application module fuzzy knowledge state definer (FuzKSD) to implement and evaluate a webbased education that performs individualised instruction on the field of programming languages (C Programming language). The system's evaluation showed that the incorporation of fuzzy sets with overlay and stereotype models significantly provides to the adaptation of the learning process to the learning movement of each learner. FuzKSD supports the students that previously understood the aspects of computer programming to save time and effort through the learning process. Although they have used more than one adaptive technique such as fuzzy logic, overlay model and stereotype in this system to enhance the adaptively and personalised e-learning, they did not give an overview of their system in general so it has hard to be clear of the structure they used.

[9] Have built a personalised remedial learning system to support learners after taking an online test evaluation. The fuzzy logic theory has been used to construct a proper learning path based on the learner's misunderstandings found in the chosen quiz. Their system will select the most suitable remedial contents for each learner based on the learning path for each learner in each concept regarding the learners' favourites. The learning path idea in this research is based on the student's misunderstanding about a particular topic, and it is different from [29] research that the learning path of each learner is based on the difficulty level of each concept. They have noticed from their study investigation that their system could be more usable if they develop the features of teaching websites, including the learning styles, and obtained online teaching contents automatically instead of adding the materials manually. Additionally, [28] have designed, implemented and evaluated DEPTHS (Design Pattern Teaching Help System) to present the student model. The student model in DEPTHS is a result of combining the stereotype and overlay modelling approaches. By using the knowledge assessment method based on fuzzy rules, which are a combination of production rules and fuzzy logic, the student model keeps updating during the learning process. The student model in the DEPTHS system has similar concepts and processes to the student's learning path in a remedial learning system that has been completed by [9]. DEPTHS is a Web-based ITS for teaching software design patterns. They have suggested upgrading their system by supporting collaborative, project-based learning to allow collaboration between the students in DEPTHS system. The updated system could integrate various current learning systems and tools such as a Learning Management System, software modelling tool, different collaboration tools and related online repositories of software DPs [31]. These integrations could develop students' learning effectiveness and performance by giving them educational services actively promoting the collaborative learning process to provide more participation in the learning process. The fuzzy knowledge model has been applied to obtain the optimised result in a proposed skill-based e-learning system [32]. This research examines the personal skill abilities and it is recommended a set of courses in adaptive curriculum and plans to the learner. However, they could run an experiment to prove their proposed system.

A review of the work above leads this research to offer a proposed system which it is postulated will provide better results by using a combination of coloured concept maps and fuzzy logic to evaluate and show the learner's knowledge level for each concept in the domain, and produce a ranked concept list of learning materials to address misconceptions in the learner's knowledge level. 


\section{SYSTEM OVERVIEW}

The Concept and Fuzzy Adaptive E-learning system (CaFAE) has been built to enhance learners' performance and understanding. The structure of the system is shown in Figure 1. There are three main components in this system; the fuzzy logic system, the coloured concept map (CCM) and the ranked concept list (RCL). Each of these components is explained in this paper in more detail due to its importance in the system to achieve the adaptation. Teachers and students can use this system, and each one has a separate user interface to interact with the system.

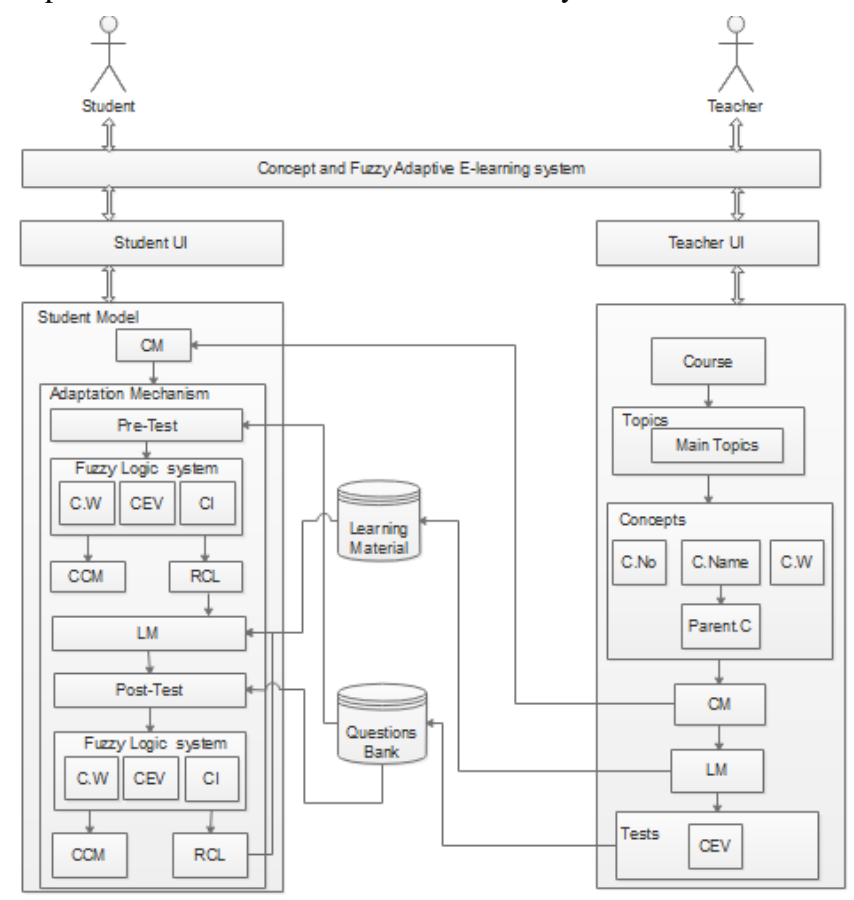

Figure 1. The architecture of CaFAE system.

\subsection{Teacher Interface}

At the beginning of using the system, teachers can login into the system within this interface, and create courses, main topics, related concepts of each topic and add learning materials (LM), and store this in the Learning Material database. To construct a concept map (CM) of the course as shown in Figure 2, teachers are able to determine the concept number (C.No), concept weight (C.W) which is explained in detail in the fuzzy logic system section, concept name (C.Name) and parent of the concepts (Parent.C) if needed. They can also create pre-and post-tests to evaluate the learner's understanding level of each concept in the topic, and store it in the Questions Bank database. To do so, concept error values (CEV), which is explained in detail in the fuzzy logic system section, must be specified when the teachers create the tests.

\subsection{Student Model}

In this model, learners are able to see the concept map of the course to have a better understanding of the course structure. Then, learners take an initial pre-test before learning the materials to evaluate their knowledge levels of each concept in the topic. A fuzzy logic system is used in the pre-test evaluation to evaluate the learner's knowledge level for each concept in the domain, and construct a coloured concept map (CCM) to show the learners their knowledge levels and produce a ranked concept list (RCL) of learning materials (LM) to address their misconceptions in the learners' understanding. Learners have to follow their ranked concept list to learn the material in different formats (text, audio and video). After using the system to learn the materials, they take a post-test to measure their abilities of understanding the concepts and are presented with a revised coloured concept to show their understanding levels of each concept. The system mechanism is described in detail in the next section.

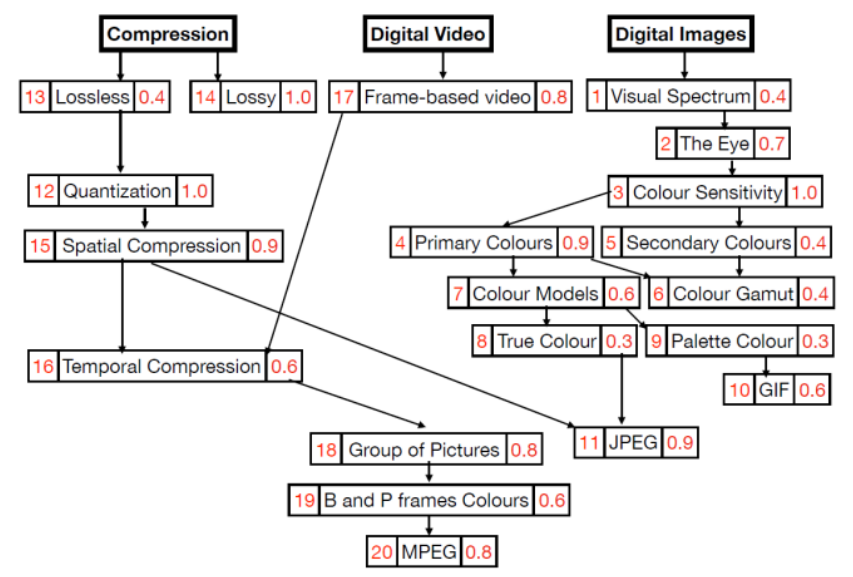

Figure 2. Course concept map for the Multimedia Design and Applications Digital Media course.

\section{ADAPTATION MECHANISM}

The adaptive process works as sequential stages in this system as follows.

\subsection{Pre-test and Post-test}

The purpose of the pre-and post-test is to assess the learners' knowledge level of the concepts for the module. To calculate both tests, two input variables are determined, Concept Weight (C.W) and Concept Error Value (CEV), and one output variable Concept Impact (CI) which are explained in detail in the fuzzy logic system section. The only one who is responsible to give these two input values in the phases of creating the concepts and tests, is the teacher of the course. The CI variable is used to give output values to be arranged in the ranked concept list (RCL) after submitting the pre-or post-test. The teachers can create an unlimited number of questions with multiple choice question type. Each question is related to one concept in the topic to determine the knowledge level of the students in each concept, and the students are allowed to choose only one choice for each question. Each choice of each question could be one of the knowledge level categories (Learned, Known, Unsatisfied known, and Unknown). Each choice has its own error value determined by the teacher.

\subsection{CCM and RCL}

After submitting the pre-or post-test, students receive two major components in this system. They can display their coloured concept map to discover their knowledge levels for each concept in the domain, and they can follow their ranked concept list based on their results of the pre-test. Therefore, we represent the knowledge level category via a colour representation of the concept map shown in Fig. 2 based on the student's answers to the pre-test and post-test. Students can follow their own learning level in terms of concepts through this map. Concepts with knowledge levels are indicated as "Learned" with green, "Known" with blue, "Unsatisfied known" with orange, "Unknown" with red. 
Students must follow their ranked concept list based on their pre-or post-test results after completing each test. They will learn the learning material based on the provided ranked list concept by concept until they complete all the concepts in the topic. After learning all the concepts, they can take a post-test to evaluate their knowledge levels of each concept in the topic.

\subsection{LM}

As we mentioned above, teachers can add the learning materials and store them into the LM database once they create the course. Learning materials could be provided in different formats (text, audio and video). Students can learn with their preferable learning style, and can choose from these learning media. They cannot choose any concept in the course to learn without first taking the pre-test. Therefore, learning materials are provided to the students based on their concept understanding and ranked concept list.

\section{FUZZY LOGIC SYSTEM}

In this system, fuzzy logic will be used to assess the learners' knowledge level for each concept in the domain, and recommend the suitable learning materials.

\subsection{Fuzzy Logic Design}

To build the fuzzy logic system [33], the problem needs to be specified and the input and output variables and their range determined, fuzzy sets is determined, fuzzy rules is constructed, and fuzzy inference is performed. Based on these tasks, we follow these following steps:

\subsubsection{Specify the problem and define the linguistic variables}

For this system, there are three main linguistic variables: concept weight C.W, concept error value $\mathrm{CEV}$ as input variables, and concept impact $\mathrm{CI}$ as an output variable. The linguistic variables are shown in Figure 3.

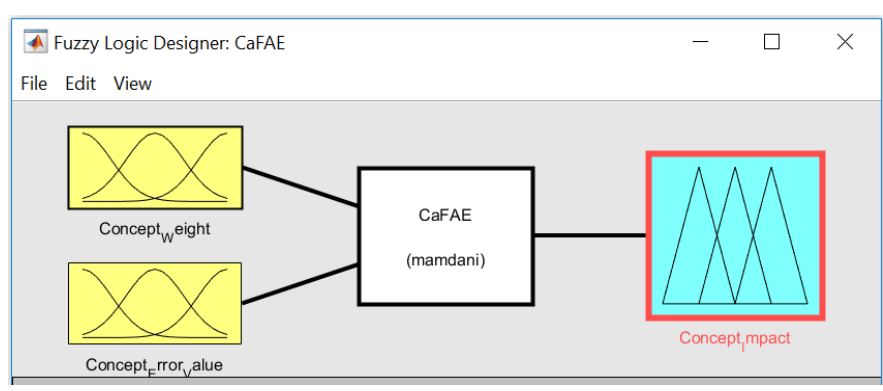

Figure 3. Linguistic variables of CaFAE system.

\subsubsection{Concept Weight (C.W)}

The concept weight variable $\mathrm{C} . \mathrm{W}$ is the first input variable, and it is considered the most important factor of the learning process environment because it determines which is the most important concept which must be understood before learning the other related concepts in the topic. In this system, each concept has its own weight which is determined by the expert of the course, and the range values of the concept weight is between ( 0.0 and 1.0). Therefore, when the concept weight reaches the value (1.0), the concept becomes the most important amongst related concepts in the topic. On the other hand, when the concept weight has a smaller value than the others, it becomes a less important concept between the related concepts. For this study, in the fuzzy logic system, there are three linguistic values Small, Medium, and Large, and each linguistic value has its range of fuzzy values $(0.0$ to 0.4$),(0.3$ to 0.8$)$ and $(0.7$ to 1.0$)$ respectively to show the importance of learning a specific concept.

\subsubsection{Concept Error Value (CEV)}

The concept error value CEV is the second input variable, and it is derived from the student's answer from the pre- or post-test in the beginning or during learning the materials of the course. Therefore, the CEV has four linguistic values Learned, Known, Unsatisfied Known, and Unknown, and each linguistic value has its own range of fuzzy values ( 0 to 25$),(20$ to 50$),(45$ to 75$)$ and (70 to 100$)$ respectively to determine the knowledge level of each concept in the domain for each student.

\subsubsection{Concept Impact (CI)}

The concept impact value is an output variable, and it is a result of the input variables C.W and CEV calculation using MATLAB Fuzzy Logic Toolbox from the MathWorks (Negnevitsky, Michael, 2005). This value will be obtained from each question. Therefore, we will have 20 concept impact values. All these values will be arranged into the ranked concepts list in different methods. Therefore, we categorise the output values based on MATLAB Fuzzy Logic Toolbox result, and CI has three linguistic values Small, Medium, and Large, and each linguistic value has its range of fuzzy values (0 to 35$),(25$ to 70$)$ and (60 to 100) respectively to be arranged in the ranked list from which students can learn the appropriate related concepts in a topic.

\subsubsection{Determine Fuzzy Sets}

A fuzzy set is a set of objects with fuzzy boundaries such as low, medium or high for concept's level of understanding. To assign a fuzzy set, the proposed system represents it as a function and then maps the elements of the set to their degree of membership. The ideal example in fuzzy sets in the proposed system is an unknown concept. The elements of its fuzzy set "unknown" are all weak or unknown concepts, but their degrees of membership are based on their levels. Standard membership functions applied in fuzzy expert systems are triangles and trapezoids.

\subsubsection{Construct Fuzzy Rules}

After defining the input and output variables with their fuzzy sets, the proposed system addresses the fuzzy rules as in Figure 4, and the only one who can build the fuzzy rules is the expert because they already know how the problem can be solved using all the linguistic variables with their terms. Fuzzy rules are used to take personal knowledge. A fuzzy rule is a conditional statement in the form (IF $\mathrm{x}$ is $\mathrm{A}$, THEN $\mathrm{y}$ is $\mathrm{B}$ ) where $\mathrm{x}$ and $\mathrm{y}$ are linguistic variables, and $A$ and $B$ are linguistic values defined by fuzzy sets. Therefore, there are three concept weights and four concept error values which produces 12 possible rules shown in Fig. 4. For example, IF the concept weight is Medium AND the concept error value is Known THEN the impact of the concept is Small.

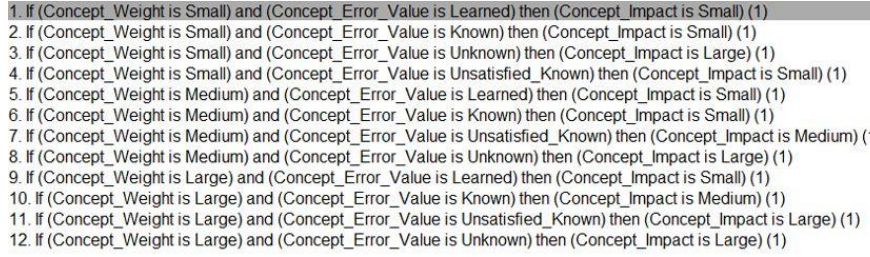

Figure 4. fuzzy rules inference using MATLAB fuzzy Logic Toolbox

\subsubsection{Performing the Fuzzy Inference}

The process of expressing the mapping from one specific input to an output by using the fuzzy sets theory is known as fuzzy inference. 
There are four steps involved in this process: fuzzification of the input variables, rule evaluation, aggregation of the rule outputs and defuzzification.

\subsubsection{Fuzzification}

Initially the crisp inputs are taken, C.W and CEV (concept weight and concept error value), and determine to what degree these inputs belong to each relevant fuzzy set. At this stage the degree of membership for the linguistic values of C.W and CEV is calculated. The C.W and CEV values are determined by the teachers when creating the tests and concepts. The teachers can offer numbers between 0.0 and 1.0 which are representative of the concept weight. Additionally, they give numbers between 0 and 100 to represent the concept error value for each of the choices during pre- or post-test creation. After the crisp inputs C.W and CEV are obtained, they are fuzzified against the appropriate linguistic fuzzy sets. Thus, each input is fuzzified over all the membership functions used by the fuzzy rules.

\subsubsection{Rule Evaluation or Inference Step}

Next, the fuzzified inputs are taken and applied to the antecedents of the fuzzy rules, displayed in Fig. 4. Each fuzzy rule has multiple antecedents, and the fuzzy operator (AND) is used to obtain a single number which represents the result of the antecedent evaluation. This number (the truth value) is subsequently applied to the consequent membership function. Via this system, the fuzzy inference uses AND operation between the C.W and CEV as input linguistic variables in the IF antecedents part, and output is used in the linguistic variable in the THEN consequent part. For evaluation of the conjunction of the rule antecedents, we apply the AND fuzzy operation intersection with min method as in (1):

$\mu A \cap B(X)=\min [\mu A(x), \mu B(x)]$

Based on (1), we examined our rules using rule number 6:

IF $\mathrm{x}$ is $\mathrm{A}$

AND y is B

is known (30)

THEN $\mathrm{z}$ is $\mathrm{C}$

small (14.9)

$\mu C(z)=\min [\mu A(x), \mu B(y)]=\min [0.5,30]=14.9$

12 rules are employed, which are based on the combinations which are possible of the two linguistic terms and the two fuzzy input variables. Every fuzzy inference rule is defined by the prior knowledge of the domain expert. Rule output variables are defined as fuzzy output variable CI, the concept impact, which includes Small, Medium, and Large as its three associated linguistic terms. The last three output variable values CI represents the concept impact levels.

\subsubsection{Aggregation of the Rule Outputs}

Aggregation is the unification output process of all rules. Rule consequent membership functions will be combined into a single fuzzy set. Therefore, the aggregation process input is the list of consequent membership functions, and the output is one fuzzy set for each of the output variables.

\subsubsection{Defuzzification}

The final stage in the fuzzy inference process is defuzzification. Fuzziness evaluates the rules, however the final output of a fuzzy system must be a crisp number. The defuzzification process input is the aggregate output fuzzy set, with a single number as the output.

\section{RANKED CONCEPT LIST}

After completing the pre-test, students receive their concept impact values derived from the questions' answers. To arrange the ranked concepts list (RCL), the priority to be the top of the list to be learned first is the highest concept impact value from all the concept impact values, and then the smaller values until the smallest value to be the last concept to be learned. However, if two or more concept impact values are equal and have the same value, we will have different methods to solve this problem, which are explained in this section.

There are two methods to arrange the ranked concepts list based on the highest value for each variable:

\subsection{First Method}

Ranked Concepts List $($ RCL) $=$ Concept Impact Value $(\mathrm{CIV}) \rightarrow$ Concept Weight (C.W) $\rightarrow$ Concept Number (C.No)

\section{Ranked Concept List arrangement:}

Input: Concept Impact $(\boldsymbol{C I})$, Concept Weight $(\boldsymbol{C}$. W $)$, Concept

Error Value $(\boldsymbol{C E} \boldsymbol{V})$, and Concept Number $(\boldsymbol{C} . \mathbf{N o})$

Output: A suitable ranked concept list $(\boldsymbol{R C L})$

Process:

1) $\boldsymbol{R C L}=[1]$

2) Find the highest concept impact value $(\boldsymbol{C I})$ from the ranked concept list $(\boldsymbol{R} \boldsymbol{C L})$ values.

3) Add the highest $\boldsymbol{C I}$ into first place in $\boldsymbol{R} \boldsymbol{C} \boldsymbol{L}$. While ( $\boldsymbol{C I}$ value < highest $\boldsymbol{C I}$ value) $\{$. Find the next highest $(\boldsymbol{C I})$ value to the head of the $\boldsymbol{R} \boldsymbol{C L}$. Add the next highest $\boldsymbol{C I}$ into the next place in $\boldsymbol{R C L}$. If $(\boldsymbol{C I}$ value $=$ highest $\boldsymbol{C I}$ value $)$

Do \{

Find the highest concept weight $\boldsymbol{C} . \boldsymbol{W}$ from the concepts that have the same $\boldsymbol{C I}$ values.

Add the highest $\boldsymbol{C}$. $\boldsymbol{W}$ into the next place in

RCL.

While $(\boldsymbol{C} . \boldsymbol{W}$ value < highest $\boldsymbol{C}$. $\boldsymbol{W}$ value $)\{$.

Find the next highest $(\boldsymbol{C} . \boldsymbol{W})$ value to the head of the $\boldsymbol{R} \boldsymbol{C L}$.

Add the next highest $\boldsymbol{C} . \boldsymbol{W}$ into the next place in $\boldsymbol{R C L}$.

else

If $(\boldsymbol{C I}$ value $=$ highest $\boldsymbol{C I}$ value $)$ and $(\boldsymbol{C} . \boldsymbol{W}$ value $=$ highest $\boldsymbol{C}$. $\boldsymbol{W}$ value)

Do \{

Find the first concept number (C.No) in the

RCL.

Add the first $\boldsymbol{C}$.No into the next place in $\boldsymbol{R} \boldsymbol{C} \boldsymbol{L}$. \} until ( $\boldsymbol{C I}$ value $\neq$ highest $\boldsymbol{C I}$ value) and

( $\boldsymbol{C} . \boldsymbol{W}$ value $\neq$ highest $\boldsymbol{C}$. $\boldsymbol{W}$ value $)$

\} until ( $\boldsymbol{C I}$ value $\neq$ highest $\boldsymbol{C I}$ value $)$

\}

else Add $\boldsymbol{C I}$ to $\boldsymbol{R} \boldsymbol{C L}$

4) Return $\boldsymbol{R C L}$.

In this method, as explained in the algorithm above the priority to be learned first is the highest concept impact value (CIV), and then followed by the smaller values, but if there are two or more concept impact values (CIV) that are equal, then we compare between the concept weights (C.W) of these equally concept impact values (CIV), and the highest concept weight value (C.W) between these concepts will be the priority to be learned first. Finally, if two or more concepts have equal concept impact values (CIV) and equal 
concept weight values (C.W), then the priority to be learned first is based on the concept number (C.No) in the ranked concepts list (RCL).

As shown in Table 1, a student follows the ranked concepts list based on "First Method RCL" from 1 to 10 in ascending order after completing his/her pre- or post-test. The student learns C.No "4" first because it has the highest concept impact (83.4) between all the concepts, and then s/he learns the following concepts that have smaller concept impact values respectively such as C.No "8" "1" and " 2 ". However, if two or more concepts have the same concept impact value (CIV) such as C.No "3", "7" and "9", they have the same CIV (16), therefore, the priority to be learned next is the highest concept weight between these concepts that have the same concept impact value (CIV). C.No " 3 " has the highest concept weight (1.0), so, the student learns it first, then C.No "7" (0.6), and C.No "9" (0.3) respectively. In case of two or more concepts have the same concept impact value (CIV) and concept weight (C.W) such as C.No " 5 " and " 6 ", they have the same CIV (15.8) and C.W (0.4), therefore, the priority to be learned next is based on the concept number (C.No) in the ranked concepts list (RCL).

Table 1. knowledge level of a student

\begin{tabular}{|c|c|c|c|c|c|l|c|}
\hline C.No & C.W & CEV & KL & CIV & CI & $\begin{array}{l}\text { FIRST } \\
\text { METHOD RCL }\end{array}$ & $\begin{array}{c}\text { Second } \\
\text { Method RCL }\end{array}$ \\
\hline 1 & 0.4 & 75 & UK & 81.9 & L & 3 & 3 \\
\hline 2 & 0.7 & 60 & USK & 48 & M & 4 & 4 \\
\hline 3 & 1.0 & 25 & K & 16 & S & 5 & 5 \\
\hline 4 & 0.9 & 55 & USK & 83.4 & L & 1 & 1 \\
\hline 5 & 0.4 & 35 & K & 15.8 & S & 8 & 8 \\
\hline 6 & 0.4 & 17 & L & 15.8 & S & 9 & 9 \\
\hline 7 & 0.6 & 45 & K & 16 & S & 6 & 6 \\
\hline 8 & 0.3 & 99 & UK & 82.6 & L & 2 & 2 \\
\hline 9 & 0.3 & 50 & USK & 16 & S & 7 & 7 \\
\hline 10 & 0.6 & 0 & L & 14.5 & S & 10 & 10 \\
\hline
\end{tabular}

\subsection{Second Method}

Ranked Concepts List $(\mathrm{RCL})=$ Concept Impact Value $(\mathrm{CIV}) \boldsymbol{\rightarrow}$ Concept Weight $(\mathrm{C} . \mathrm{W}) \rightarrow$ Concept Error Value $(\mathrm{CEV}) \rightarrow$ Concept Number (C.No)

\section{Ranked Concept List arrangement:}

Input: Concept Impact $(\boldsymbol{C I})$, Concept Weight $(\boldsymbol{C}$. $\boldsymbol{W})$, Concept

Error Value $(\boldsymbol{C E} \boldsymbol{V})$, and Concept Number $(\boldsymbol{C} . \boldsymbol{N o})$

Output: A suitable ranked concept list $(\boldsymbol{R C} \boldsymbol{L})$

Process:

1) $\boldsymbol{R C L}=[1]$

2) Find the highest concept impact value $(\boldsymbol{C I})$ from the ranked concept list $(\boldsymbol{R} \boldsymbol{C L})$ values.

3) Add the highest $\boldsymbol{C I}$ into first place in $\boldsymbol{R} \boldsymbol{C L}$.

While ( $\boldsymbol{C I}$ value $<$ highest $\boldsymbol{C I}$ value) $\{$.

Find the next highest $(\boldsymbol{C I})$ value to the head of the $\boldsymbol{R} \boldsymbol{C L}$. Add the next highest $\boldsymbol{C I}$ into the next place in $\boldsymbol{R} \boldsymbol{C L}$. If $(\boldsymbol{C I}$ value $=$ highest $\boldsymbol{C I}$ value $)$

Do \{

Find the highest concept weight $\boldsymbol{C} . \boldsymbol{W}$ from the concepts that have the same $\boldsymbol{C I}$ values.

RCL.

Add the highest $\boldsymbol{C}$. $\boldsymbol{W}$ into the next place in

While $(\boldsymbol{C} . \boldsymbol{W}$ value $<$ highest $\boldsymbol{C} . \boldsymbol{W}$ value $)\{$.
Find the next highest $(\boldsymbol{C} . \boldsymbol{W})$ value to the head of the $\boldsymbol{R} \boldsymbol{C L}$.

Add the next highest $\boldsymbol{C}$. $\boldsymbol{W}$ into the next place in $R C L$

else

If $(\boldsymbol{C I}$ value $=$ highest $\boldsymbol{C I}$ value $)$ and $(\boldsymbol{C} . \boldsymbol{W}$ value $=$ highest $\boldsymbol{C} . \boldsymbol{W}$ value)

Do \{

Find the highest concept error value $\boldsymbol{C E} \boldsymbol{V}$ from the concepts that have the same $\boldsymbol{C I}$ values and $\boldsymbol{C}$. $\boldsymbol{W}$ values.

Add the highest $\boldsymbol{C E} \boldsymbol{V}$ into the next place in

RCL.

While ( $\boldsymbol{C} \boldsymbol{E} \boldsymbol{V}$ value < highest $\boldsymbol{C} \boldsymbol{E} \boldsymbol{V}$ value $)\{$.

Find the next highest $(\boldsymbol{C} \boldsymbol{E} \boldsymbol{V})$ value to the head of the $\boldsymbol{R C L}$.

Add the next highest $\boldsymbol{C E} \boldsymbol{V}$ into the next place

in $R C L$

else

If ( $\boldsymbol{C I}$ value $=$ highest $\boldsymbol{C I}$ value $)$ and $(\boldsymbol{C} . \boldsymbol{W}$ value $=$ highest $\boldsymbol{C} . \boldsymbol{W}$ value $)$ and $(\boldsymbol{C} \boldsymbol{E} \boldsymbol{V}$ value $=$ highest $\boldsymbol{C} \boldsymbol{E} \boldsymbol{V}$ value)

Do \{

Find the first concept number (C.No) in the

RCL.

Add the first $\boldsymbol{C}$.No into the next place in $\boldsymbol{R} \boldsymbol{C L}$. \} until ( $\boldsymbol{C I}$ value $\neq$ highest $\boldsymbol{C I}$ value) and ( $\boldsymbol{C}$. $\boldsymbol{W}$ value $\neq$ highest $\boldsymbol{C} . \boldsymbol{W}$ value $)$ and ( $\boldsymbol{C} \boldsymbol{V}$ value $\neq$ highest $\boldsymbol{C} \boldsymbol{E} \boldsymbol{V}$ value)

\} until ( $\boldsymbol{C I}$ value $\neq$ highest $\boldsymbol{C I}$ value) and ( $\boldsymbol{C} . \boldsymbol{W}$ value $\neq$ highest $\boldsymbol{C}$. $\boldsymbol{W}$ value)

\} until ( $\boldsymbol{C I}$ value $\neq$ highest $\boldsymbol{C I}$ value)

\}

else Add $\boldsymbol{C I}$ to $\boldsymbol{R} \boldsymbol{C} \boldsymbol{L}$

\section{4) Return $\boldsymbol{R C L}$.}

In this method, the priority to be learned first is the highest concept impact value (CIV), and then followed by the smaller values, but if there are two or more concept impact values (CIV) that are equal, then we compare between the concept weights (C.W) of these equally concept impact values (CIV), and the highest concept weight value (C.W) between these concepts will be the priority to be learned first. However, if two or more concepts have equal concept impact values (CIV) and equal concept weight values (C.W), then we compare between the concept error values (CEV) of these equally concept impact values (CIV) and equal concept weights (C.W), and the highest concept error value (CEV) between these concepts will be the priority to be learned first. Finally, if two or more concepts have equal concept impact values (CIV), equal concept weight values (C.W) and equal concept error values (CEV), then the priority to be learned first is based on the concept number (C.No) in the ranked concepts list (RCL).

As shown in Table 1, Second Method RCL has the same process and order in First Method, but there is different comparison in case of two or more concepts have the same CIV and C.W such as C.No " 5 " and "6". They have the same CIV (15.8) and C.W (0.4), therefore, the priority to be learned next is the highest concept error value (CEV) between these concepts that have the same concept impact value (CIV) and concept weight (C.W). C.No " 5 " has the highest concept error value (35), so, the student learns it first, then C.No "6" (17) next. 


\section{Conclusion}

This paper presents an adaptive e-learning system called Concept and Fuzzy Adaptive E-learning (CaFAE). CaFAE has been developed to evaluate and show the learner's knowledge level for each concept in the domain, and produce a ranked concept list of learning materials to identify misconceptions in the learner's knowledge level. A coloured concept map has been designed to show the student's knowledge level for each concept in the topic, and to increase participants' overall learning level and effectiveness. Fuzzy logic is used to assess the learners' knowledge level for each concept in the domain, and recommend the suitable learning materials using a suitable ranked concept list.

For future work we are planning to run a pilot study in a real class environment to test the system. Although other researchers have designed systems using fuzzy logic or concept maps, and run their experiments on programming languages, the proposed system has several novel components and will be tested as part of a technical digital media course with cohorts of students who will study this course at an undergraduate level.

\section{REFERENCES}

[1] Akscyn, R.M., D.L. McCracken, and E.A. Yoder, KMS: a distributed hypermedia system for managing knowledge in organizations. Communications of the ACM, 1988. 31(7): p. 820835 .

[2] Moore, J.L., C. Dickson-Deane, and K. Galyen, $e$ Learning, online learning, and distance learning environments: Are they the same? The Internet and Higher Education, 2011. 14(2): p. 129-135.

[3] Liaw, S.-S., Investigating students' perceived satisfaction, behavioral intention, and effectiveness of e-learning: A case study of the Blackboard system. Computers \& Education, 2008. 51(2): p. 864-873.

[4] Conlan, O., D. Dagger, and V. Wade. Towards a standards-based approach to e-Learning personalization using reusable learning objects. in Proc. of World Conference on ELearning, E-Learn. 2002.

[5] Pechenizkiy, M., et al. Mining the student assessment data: Lessons drawn from a small scale case study. in Educational Data Mining 2008. 2008.

[6] Khemaja, M. and A. Taamallah, Towards Situation Driven Mobile Tutoring System for Learning Languages and Communication Skills: Application to Users with Specific Needs. Educational Technology \& Society, 2016. 19(1): p. 113-128.

[7] Klašnja-Milićević, A., et al., Personalization and Adaptation in E-Learning Systems, in E-Learning Systems. 2017, Springer. p. 21-25.

[8] Vanides, J., et al., Concept maps. Science Scope, 2005. 28(8): p. 27-31.

[9] Hsieh, T.-C., M.-C. Lee, and C.-Y. Su, Designing and implementing a personalized remedial learning system for enhancing the programming learning. Educational Technology \& Society, 2013. 16(4): p. 32-46.

[10] Stansfield, J.L., Wumpus Advisor I. A First Implementation of a Program That Tutors Logical and Probabilistic Reasoning Skills. AI Memo 381. 1976.

[11] Rich, E., User modeling via stereotypes. Cognitive science, 1979. 3(4): p. 329-354.

[12] Liebowitz, J., Expert systems: A short introduction. Engineering Fracture Mechanics, 1995. 50(5): p. 601-607.

[13] Ben-Gal, I., Bayesian networks. Encyclopedia of statistics in quality and reliability, 2007.
[14] Birjali, M., A. Beni-Hssane, and M. Erritali, A novel adaptive e-learning model based on Big Data by using competencebased knowledge and social learner activities. Applied Soft Computing, 2018. 69: p. 14-32.

[15] Dogan, B. and E. Dikbiyı, OPCOMITS: Developing an adaptive and intelligent web based educational system based on concept map model. Computer Applications in Engineering Education, 2016. 24(5): p. 676-691.

[16] Violante, M.G. and E. Vezzetti, Design of web-based interactive $3 D$ concept maps: A preliminary study for an engineering drawing course. Computer Applications in Engineering Education, 2015. 23(3): p. 403-411.

[17] Lin, Y.S., et al., Effects of concept map extraction and a test-based diagnostic environment on learning achievement and learners' perceptions. British Journal of Educational Technology, 2016. 47(4): p. 649-664.

[18] Awati, M.A.S. and A. Dixit, Automated Evaluation Framework for Student Learning using Concept Maps. 2017.

[19] Zadeh, L.A., Fuzzy sets. Information and control, 1965. 8(3): p. 338-353.

[20] Zadeh, L.A., Fuzzy logic= computing with words. IEEE transactions on fuzzy systems, 1996. 4(2): p. 103-111.

[21] Hájek, P., What is mathematical fuzzy logic. Fuzzy sets and systems, 2006. 157(5): p. 597-603.

[22] Zadeh, L.A., Making computers think like people [fuzzy set theory]. IEEE spectrum, 1984. 21(8): p. 26-32.

[23] Yen, J., L. Wang, and C.W. Gillespie, Improving the interpretability of TSK fuzzy models by combining global learning and local learning. IEEE Transactions on fuzzy Systems, 1998. 6(4): p. 530-537.

[24] Zimmermann, H.-J., Fuzzy set theory-and its applications. 2011: Springer Science \& Business Media.

[25] Chrysafiadi, K. and M. Virvou, Evaluating the integration of fuzzy logic into the student model of a web-based learning environment. Expert Systems with Applications, 2012. 39(18): p. 13127-13134.

[26] Chrysafiadi, K. and M. Virvou, A knowledge representation approach using fuzzy cognitive maps for better navigation support in an adaptive learning system. SpringerPlus, 2013. 2(1): p. 81.

[27] Chrysafiadi, K. and M. Virvou, Fuzzy logic for adaptive instruction in an e-learning environment for computer programming. IEEE transactions on Fuzzy Systems, 2015. 23(1): p. 164-177.

[28] Jeremić, Z., J. Jovanović, and D. Gašević, Student modeling and assessment in intelligent tutoring of software patterns. Expert Systems with Applications, 2012. 39(1): p. 210222.

[29] Zafar, A. and I. Albidewi, Evaluation study of eLGuide: A framework for adaptive e-learning. Computer Applications in Engineering Education, 2015. 23(4): p. 542-555.

[30] Kirkpatrick, D.L., Techniques for evaluating training programs. Training and development journal, 1979.

[31] Jeremić, Z., J. Jovanović, and D. Gašević, Evaluating an intelligent tutoring system for design patterns: The DEPTHS experience. Educational Technology \& Society, 2009. 12(2): p. 111-130.

[32] C., G. and R. V., Skill-based education through fuzzy knowledge modeling for e-learning. Computer Applications in Engineering Education, 2018. 26(2): p. 393-404.

[33] Negnevitsky, M., Artificial intelligence: a guide to intelligent systems. 2005: Pearson Education. 


\section{Columns on Last Page Should Be Made As Close As Possible to Equal Length}

\section{Authors' background}

\begin{tabular}{|l|l|l|l|}
\hline Your Name & Title* & $\begin{array}{l}\text { Research } \\
\text { Field }\end{array}$ & Personal website \\
\hline $\begin{array}{l}\text { Mesfer Al } \\
\text { Duhayyim }\end{array}$ & $\begin{array}{l}\text { PhD } \\
\text { candidate }\end{array}$ & $\begin{array}{l}\text { Adaptive E- } \\
\text { learning }\end{array}$ & http://www.sussex.ac.uk/informatics/people/peoplelists/person/389243 \\
\hline $\begin{array}{l}\text { Paul } \\
\text { Newbury }\end{array}$ & $\begin{array}{l}\text { senior } \\
\text { lecture }\end{array}$ & $\begin{array}{l}\text { Technology } \\
\text { Enhanced } \\
\text { Learning, } \\
\text { Virtual } \\
\text { Prototyping }\end{array}$ & http://www.sussex.ac.uk/profiles/126552 \\
\hline
\end{tabular}

${ }^{*}$ This form helps us to understand your paper better, the form itself will not be published.

${ }^{*}$ Title can be chosen from: master student, Phd candidate, assistant professor, lecture, senior lecture, associate professor, full professor 